\title{
Inhibition of protein kinase CK2 facilitates cellular senescence by inhibiting the expression of HO-1 in articular chondrocytes
}

\author{
KANG MI KIM ${ }^{1}$, DONG HYUN SOHN ${ }^{1}$, KOANHOI KIM ${ }^{2}$ and YOUNG CHUL PARK ${ }^{1}$ \\ Departments of ${ }^{1}$ Microbiology and Immunology and ${ }^{2}$ Pharmacology, \\ Pusan National University School of Medicine, Yangsan, Gyeongnam 50612, Republic of Korea
}

Received June 21, 2018; Accepted November 27, 2018

DOI: $10.3892 / \mathrm{ijmm} .2018 .4016$

\begin{abstract}
Protein kinase casein kinase 2 (CK2) is important in the regulation of cell proliferation and death, even under pathological conditions. Previously, we reported that CK2 regulates the expression of heme oxygenase-1 (HO-1) in stress-induced chondrocytes. In the present study, it was shown that CK2 is involved in the dedifferentiation and cellular senescence of chondrocytes. Treatment of primary articular chondrocytes with CK2 inhibitors, 4,5,6,7-terabromo-2-azabenzimidazole (TBB) or 5,6-dichlorobenzimidazole 1- $\beta$-D-ribofuranoside (DRB), induced an increase in senescence-associated $\beta$-galactosidase (SA- $\beta$-gal) staining. In addition, TBB reduced the expression of type II collagen and stimulated the accumulation of $\beta$-catenin, phenotypic markers of chondrocyte differentiation and dedifferentiation, respectively. It was also observed that the abrogation of CK 2 activity by CK 2 small interfering RNA induced phenotypes of chondrocyte senescence. The association between HO-1 and cellular senescence was also examined in CK2 inhibitor-treated chondrocytes. Pretreatment with 3-morpholinosydnonimine hydrochloride, an inducer of the HO-1 expression, or overexpression of the HO-1 gene significantly delayed chondrocyte senescence. These results show
\end{abstract}

Correspondence to: Dr Dong Hyun Sohn and Dr Young Chul Park, Department of Microbiology and Immunology, Pusan National University School of Medicine, 49 Busandaehak-ro, Mulgeum-eup, Yangsan, Gyeongnam 50612, Republic of Korea

E-mail:dhsohn@pusan.ac.kr

E-mail: ycpark@pusan.ac.kr

Abbreviations: CK2, protein kinase casein kinase 2; TBB, 4,5,6,7-terabromo-2-azabenzimidazole; DRB, 5,6-dichlorobenzimidazole 1- $\beta$-D-ribofuranoside; SA- $\beta$-gal, senescence-associated $\beta$-galactosidase; HO-1, heme oxygenase-1; SIN-1, 3-morpholinosydnonimine hydrochloride; ECM, extracellular matrix; ROS, reactive oxygen species; OA, osteoarthritis

Key words: protein kinase casein kinase 2, chondrocytes, senescence-associated $\beta$-galactosidase, cellular senescence, heme oxygenase-1 that CK2 is associated with chondrocyte differentiation and cellular senescence and that this is due to regulation of the expression of HO-1. Furthermore, the findings suggest that CK2 is crucial as an anti-aging factor during chondrocyte senescence.

\section{Introduction}

The degradation of articular cartilage is a major characteristic of several arthritic diseases, including osteoarthritis (OA) and rheumatoid arthritis (RA), which eventually result in joint destruction $(1,2)$. The biosynthetic activities of chondrocytes contribute to the stability of articular cartilage (3-5), which contains extracellular matrix (ECM) molecules that confer mechanical elasticity. By contrast, chondrocyte dysfunction enhances cartilage ECM degradation and cartilage degeneration $(6,7)$. Chondrocyte senescence is also important in articular cartilage degeneration (8), as it is associated with declines in chondrocyte numbers and contributes to the development of arthritic diseases $(9,10)$. It has been established that senescent chondrocytes accumulate in the articular cartilages of patients with arthritic diseases (11-13), however, the factors that govern chondrocyte decisions regarding senescence remain to be fully elucidated.

Protein kinase casein kinase 2 (CK2) is a constitutively active and highly conserved serine/threonine protein kinase consisting of two catalytic ( $\alpha$ and/or $\left.\alpha^{\prime}\right)$ and two regulatory $(\beta)$ subunits (14). CK2 is ubiquitously expressed in subcellular compartments in all eukaryotes and phosphorylates over 300 substrates (15), and regulates several different metabolic events as a result. Several studies have demonstrated the links between CK2 and cell growth $(16,17)$, transformation (18) and apoptosis (19), and between CK2 and inflammatory diseases $(16,20)$. However, despite research efforts on various aspects of CK2, its function in chondrocytes is only partly understood. Previously, CK2 was reported to be involved in the regulation of rat articular chondrocyte apoptosis by phosphorylating certain apoptosis-related factors (21). In another study, it was suggested that inhibition of the activity of CK2 facilitates tumor necrosis factor-mediated chondrocyte death through apoptosis, and that the activity of CK2 is downregulated in the chondrocytes of aged articular cartilage (22).

In our previous study, it was shown that CK2 is associated with the expression of heme oxygenase-1 (HO-1) in articular 
chondrocytes (23). In the present study, it was observed that inhibiting the activity of CK2 induces the senescence of primary articular chondrocytes. In addition, it was found that CK2 inhibition-mediated senescence is associated with regulation of the expression of HO-1 in chondrocytes.

\section{Materials and methods}

Reagents. The 4,5,6,7-terabromo-2-azabenzimidazole (TBB), 5,6-dichlorobenzimidazole 1- $\beta$-D-ribofuranoside (DRB), 3-morpholinosydnonimine hydrochloride (SIN-1), protease inhibitor cocktail, trypan blue solution (0.4\%), 5-bromo4-chloroindol-3-indolyl $\beta$-D-galactopyranoside, potassium ferrocyanide, and potassium ferricyanide were purchased from Sigma-Aldrich; Merck KGaA (Darmstadt, Germany). Fetal bovine serum (FBS), Dulbecco's modified Eagle's medium (DMEM) and other culture reagents were purchased from GE Healthcare Life Sciences (Logan, UT, USA). Anti-CK2 (sc-373894), HO-1 (sc-10789), type II collagen (sc-52658), $\beta$-catenin (sc-7199), and $\beta$-actin (sc-1616-R) antibodies were obtained from Santa Cruz Biotechnology, Inc. (Santa Cruz, CA, USA). Secondary horseradish peroxidase (HRP)-conjugated antibodies (anti-rabbit, NA934; anti-mouse, NA931) and the enhanced chemiluminescence (ECL) western blotting kit were obtained from GE Healthcare Life Sciences.

Animals and cell culture of articular chondrocytes. Five-week-old female Sprague-Dawley rats were purchased from Samtako Bio Korea, Co. (Osan, Korea). All animal experiments and protocols were approved by the Pusan National University Institutional Animal Care and Use Committee (Miryang, Korea) and performed in accordance with the institutional and national guidelines for the care and use of laboratory animals. The knee joint cartilages were collected shortly upon arrival, however if necessary, the rats were housed under controlled conditions at $23 \pm 2^{\circ} \mathrm{C}$ and $50 \pm 10 \%$ humidity on a $12 \mathrm{~h} \mathrm{light/dark} \mathrm{cycle} \mathrm{and} \mathrm{had} \mathrm{free}$ access to a standard chow diet and water.

A total of 30 rats were used in the present study and their weight range was $110-130 \mathrm{~g}$. To obtain primary articular chondrocytes, the knee joint cartilages from three rats per experiment were cut into $\sim 1-\mathrm{mm}^{3}$ sections, and the samples were incubated for $1 \mathrm{~h}$ at $37^{\circ} \mathrm{C}$ with $0.2 \%$ type II collagenase in DMEM. Primary chondrocytes were collected by centrifugation (300 x g; $5 \mathrm{~min}$; room temperature) and resuspended in DMEM supplemented with $10 \%$ (v/v) heat-inactivated FBS and antibiotics (50 U/ml penicillin, $50 \mu \mathrm{g} / \mathrm{ml}$ streptomycin) at $37^{\circ} \mathrm{C}$ in a $5 \% \mathrm{CO}_{2} / 95 \%$ air atmosphere. The cells were then plated on culture dishes at $5 \times 10^{4}$ cells $/ \mathrm{cm}^{2}$ and cultured until confluent for $\sim 4$ days (medium was replaced every 2 days during culture). Following subculture (the cells were designated as passage 1, which was used for all experiments), cells were plated at a density of $1 \times 10^{5}$ cells $/ \mathrm{cm}^{2}$ and cultured for $24 \mathrm{~h}$ in $37^{\circ} \mathrm{C}$ incubator under a humidified atmosphere conditions with $5 \% \mathrm{CO}_{2}$. Then, the cells were treated with various concentrations $(2,10$, and $50 \mu \mathrm{M})$ of TBB or DRB in the presence or absence of SIN-1 for $48 \mathrm{~h}$. The cell viabilities were determined using a trypan blue exclusion assay with a hemocytometer.
Senescence-associated $\beta$-galactosidase (SA- $\beta$-gal) staining assay. The cells were washed in phosphate-buffered saline (PBS), fixed for $5 \mathrm{~min}$ at room temperature in $0.2 \%$ glutaraldehyde $/ 2 \%$ formaldehyde, washed with PBS, and incubated with SA- $\beta$-gal staining solution $[1 \mathrm{mg} / \mathrm{ml} 5$-bromo-4-chloroindol-3-indolyl $\beta$-D-galactopyranoside, $40 \mathrm{mM}$ citrate/sodium phosphate buffer ( $\mathrm{pH}$ 6.0), $5 \mathrm{mM}$ potassium ferrocyanide, $5 \mathrm{mM}$ potassium ferricyanide, $150 \mathrm{mM} \mathrm{NaCl}$, and $2 \mathrm{mM}$ $\mathrm{MgCl}_{2}$ ] for $12 \mathrm{~h}$ at $37^{\circ} \mathrm{C}$. The percentages of senescent cells were determined by counting the numbers of blue-stained cells per 200 cells in randomly selected areas at x 20 magnification under an optical microscope.

Western blot analysis. The cells were lysed in ice-cold RIPA buffer with protease inhibitors, and protein concentrations in the whole cell lysates were determined using a colorimetric method with bicinchoninic acid protein dye reagent (Pierce; Thermo Fisher Scientific, Inc., Waltham, MA, USA). Subsequently, the proteins (20 $\mu \mathrm{g})$ were loaded onto $12 \%$ SDS-polyacrylamide gels, electrophoresed, and transferred onto nitrocellulose membranes using an electroblotting apparatus (Bio-Rad Laboratories, Inc., Richmond, CA, USA). Following blocking with 5\% skim milk in TBS-T, the membranes were incubated with primary antibodies targeting CK2 $\alpha$ (1:250), HO-1 (1:250), type II collagen (1:250), $\beta$-catenin (1:250), and $\beta$-actin (1:500) at $4^{\circ} \mathrm{C}$ overnight, washed with TBS-T, incubated with HRP-conjugated secondary antibodies $(1: 2,000)$ at room temperature for $2 \mathrm{~h}$, and developed using the ECL detection system. The blots were visualized and quantified using a LAS-3000 Luminescent Image Analyzer (FujiFilm, Tokyo, Japan). $\beta$-actin was used as an internal loading control.

Measurement of CK2 kinase activity. The activity of CK2 was measured in cell lysates using a CK2 kinase assay kit (CycLex Co., Ltd., Ina, Japan). Briefly, the purified recombinant $\mathrm{CK} 2$ (for standard curve) and cell lysates ( $2 \times 10^{5}$ cells each) from CK2 $\alpha$ small interfering (si)RNA or SCR-transfected cells were added to the wells of a CK2 substrate (recombinant p53)-precoated plate. Following the addition of kinase reaction buffer, the wells were washed and incubated with HRP-conjugated detection antibody at room temperature for $30 \mathrm{~min}$. The phosphorylation activities were assessed by adding tetramethylbenzidine and measuring the absorbances at $450 \mathrm{~nm}$. All quantifications were performed in triplicate.

Transfection of $C K 2 \alpha$ siRNA. To knock down CK2 gene expression, a 21-nucleotide RNA duplex containing 3'-dTdT overhangs (GE Healthcare Dharmacon, Inc., Lafayette, $\mathrm{CO}$, USA) was used to target rat $\mathrm{CK} 2 \alpha$ mRNA (sequence, 5'-CTGGGTGGGTGTCTCATTCAA-3'). At $24 \mathrm{~h}$ after plating, the chondrocytes at $\sim 70 \%$ confluence were transfected with $30 \mathrm{nM}$ of CK $2 \alpha$ or negative control siRNA in wells using DharmaFECT ${ }^{\circledR}$ Duo transfection reagent, according to the manufacturer's protocol.

Preparation of HO-1 expression vector and transfection. The pcDNA3 mammalian cell expression vector containing full-length rat HO-1 cDNA was used as previously 
A

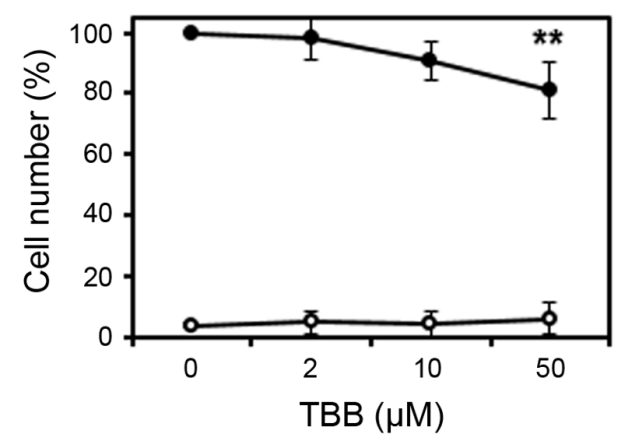

C
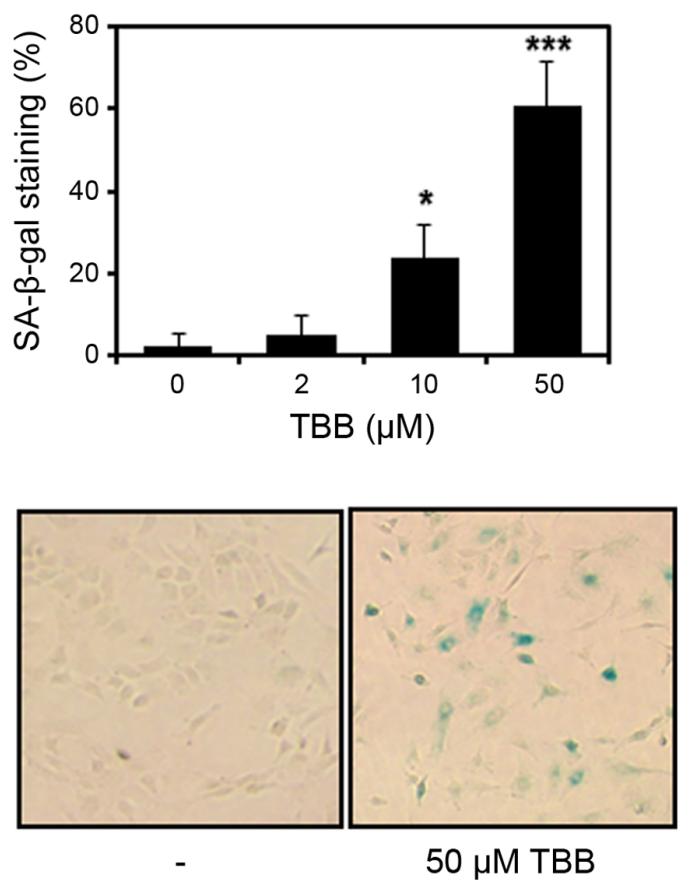

B

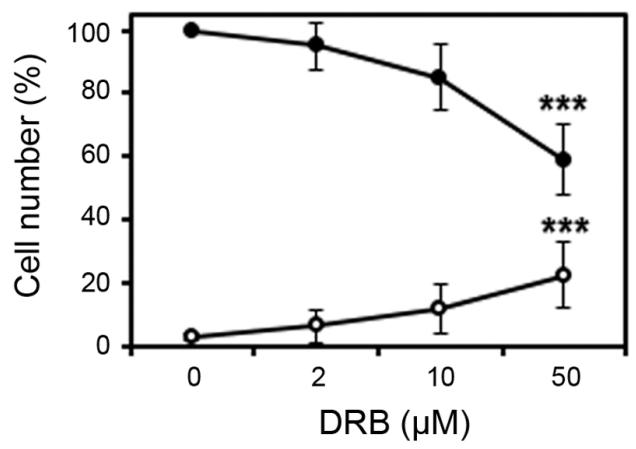

D

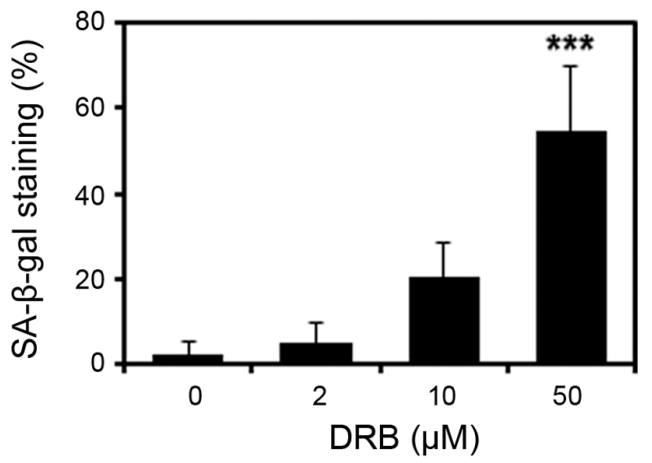

Figure 1. Effect of CK2 inhibitors on chondrocyte senescence. Primary rat articular chondrocytes were treated with different concentrations $(2,10$ and $50 \mu \mathrm{M}$ ) of TBB or DRB for $48 \mathrm{~h}$. Cells treated with (A) TBB and (B) DRB were assessed for viability using the trypan blue dye exclusion method (closed circles represent live cells, open circles represent dead cells). Values are shown as the percentages of control cells. Chondrocyte senescence was measured in cells treated with (C) TBB and (D) DRB using the SA- $\beta$-gal assay. Percentages of SA- $\beta$-gal-positive cells were calculated from numbers of blue-stained cells per 200 cells in randomly selected areas. Representative images were captured at x 20 magnification. The P-value was calculated by one-way analysis of variance followed by Dunnett's post hoc test. ${ }^{*} \mathrm{P}<0.05,{ }^{* *} \mathrm{P}<0.01$ and ${ }^{* * *} \mathrm{P}<0.001$ vs. $0 \mu \mathrm{M}$ of TBB or DRB. CK2, protein kinase casein kinase 2 ; TBB, 4,5,6,7-terabromo-2-azabenzimidazole; DRB, 5,6-dichlorobenzimidazole 1- $\beta$-D-ribofuranoside; SA- $\beta$-gal, senescence-associated $\beta$-galactosidase.

described (24). The chondrocytes $\left(1 \times 10^{5}\right.$ cells $\left./ \mathrm{cm}^{2}\right)$ were transfected with $10 \mu \mathrm{g}$ of the HO-1 construct or control plasmid complexed with Lipofectamine 2000 reagent (Invitrogen; Thermo Fisher Scientific, Inc.), incubated for $24 \mathrm{~h}$ in a $37^{\circ} \mathrm{C}$ incubator, placed in fresh medium, and then treated with TBB. The cells were also analyzed to monitor the expression level of HO-1.

Statistical analysis. All experiments were repeated at least three times. Student's t-test (two-tailed) or one-way analysis of variance were used to analyze the data by using GraphPad Prism software (GraphPad Software, Inc., La Jolla, CA, USA). Dunnett's or Tukey's honest significant difference post hoc tests were used. The results are presented as the mean \pm standard deviation, and $\mathrm{P}<0.05$ was considered to indicate a statistically significant difference.

\section{Results}

$T B B$ and DRB induces primary rat chondrocyte senescence. To determine whether the activity of CK2 is associated with cellular senescence in chondrocytes, the present study first examined the effect of TBB and DRB (pharmacological inhibitors of CK2) in primary rat chondrocytes. Isolated cells were treated with various concentrations $(2,10$ and $50 \mu \mathrm{M})$ of TBB or DRB for $48 \mathrm{~h}$ and then subjected to trypan blue dye exclusion assay and SA- $\beta$-gal staining assay. As shown in Fig. 1, TBB marginally reduced cell proliferation without affecting cell viability and significantly increased SA- $\beta$-gal staining compared with the untreated controls. The DRB-treated chondrocytes exhibited significant decreases in cell proliferation, although DRB also increased cellular senescence of chondrocytes (Fig. 1A-D). Therefore, TBB was only used as 


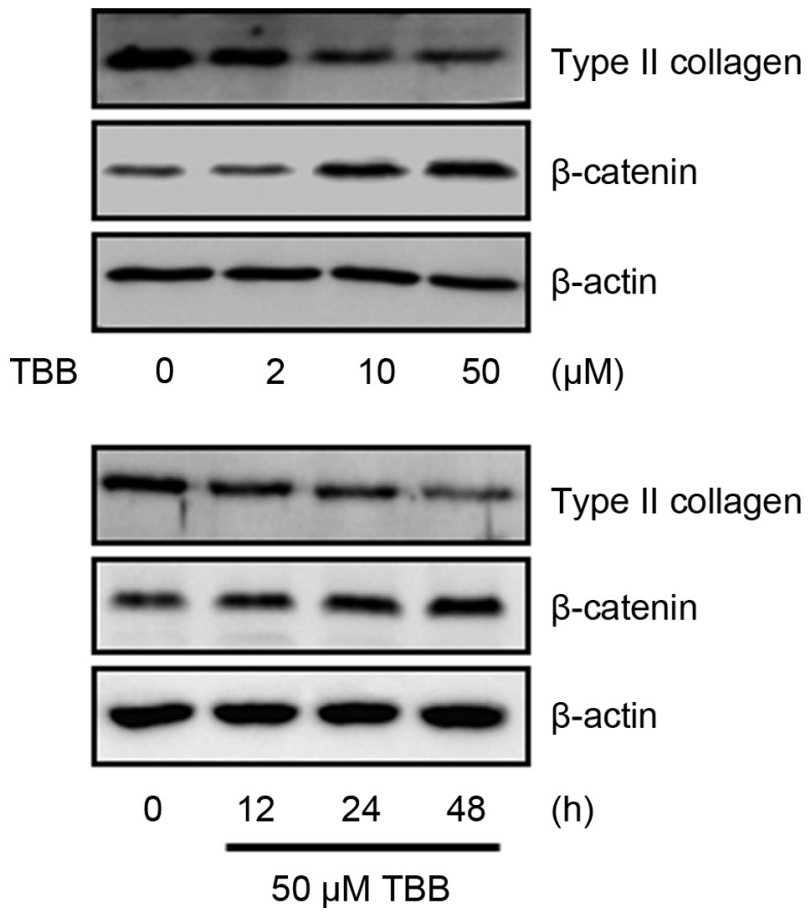

Figure 2. Effect of TBB on the expression of chondrocyte phenotypic markers. Primary chondrocytes were treated with different concentrations of TBB for $48 \mathrm{~h}$ and for different time intervals, as indicated. Whole cell lysates were analyzed by western blotting using specific antibodies against type II collagen and $\beta$-catenin. $\beta$-actin was used as an internal control. TBB, 4,5,6,7-terabromo-2-azabenzimidazole.

a specific inhibitor to downregulate the activity of CK2 in further experiments. Subsequently, the effect of TBB on the expression of phenotypic markers were examined, including type II collagen and $\beta$-catenin, which regulate chondrocyte functions. The primary chondrocytes were treated with different concentrations $(2,10$ and $50 \mu \mathrm{M})$ of TBB and for different time intervals (12, 24 or 48 h). As shown in Fig. 2, TBB significantly suppressed the expression of type II collagen and enhanced the expression of $\beta$-catenin. These results show that TBB induced changes in the expression of phenotypic markers of the differentiation and dedifferentiation of primary chondrocytes.

Specific inhibition of the activity of CK2 enhances chondrocyte senescence. To confirm that the activity of CK2 is associated with chondrocyte senescence, CK2 was knocked down by transfecting chondrocytes with a CK2 siRNA duplex or a non-specific control for $24 \mathrm{~h}$ and then incubating the cells for a further $48 \mathrm{~h}$. Transfection was found to effectively inhibit CK2 enzyme activity and to significantly reduce the protein levels of CK2 in chondrocytes (Fig. 3A and B). Transfection with control siRNA did not affect SA- $\beta$-gal staining significantly compared with the non-transfected controls. CK2 knockdown significantly increased SA- $\beta$-gal staining (Fig. 3C), and reduced the protein levels of type II collagen and enhanced $\beta$-catenin in chondrocytes. These observations suggest that CK2 activity is required to maintain chondrocyte phenotype.

SIN-1 modulates TBB-induced cellular senescence in chondrocytes. Subsequently, the present study investigated the

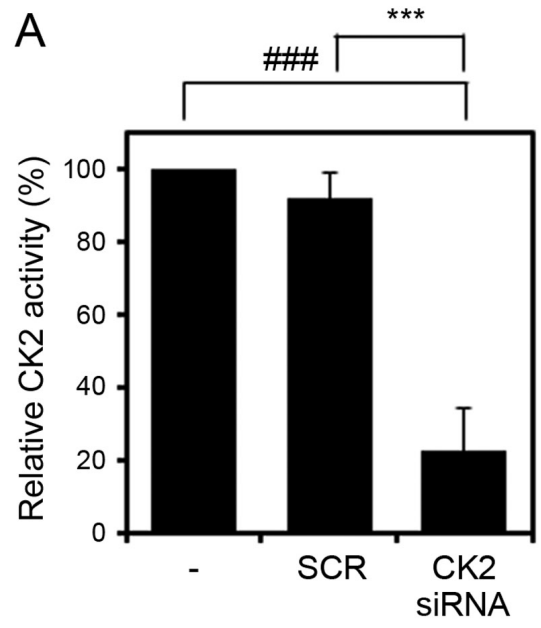

B
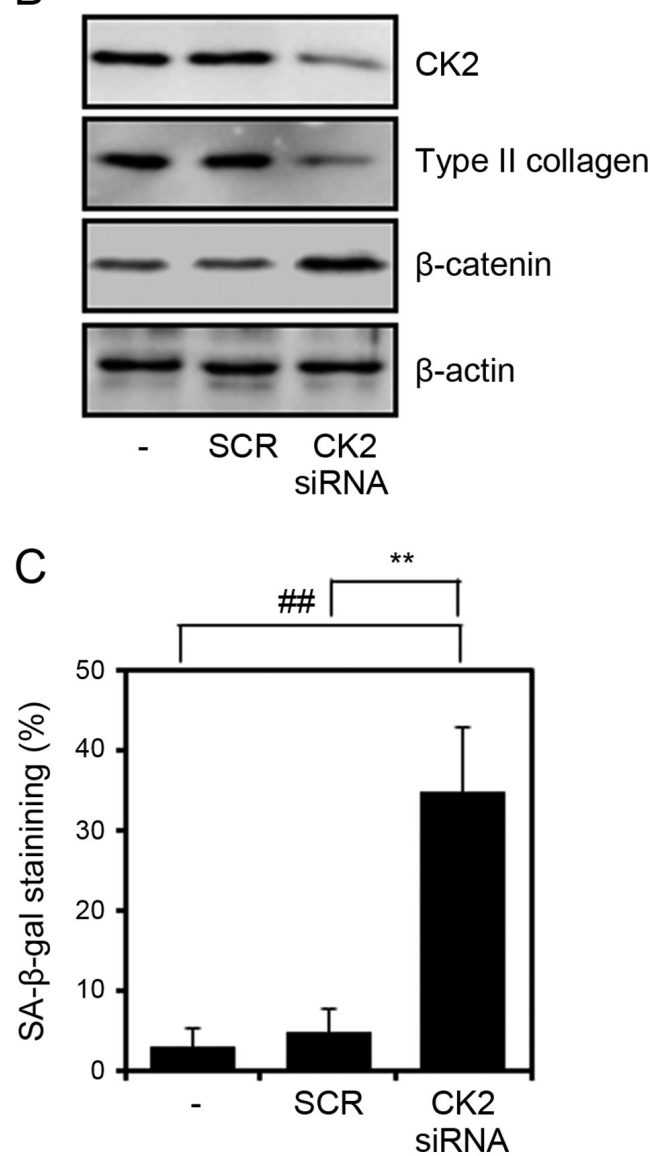

Figure 3. Effect of CK2 inhibition on chondrocyte senescence. Cells were transfected with CK2 $\alpha$ siRNA, incubated for $48 \mathrm{~h}$, and subjected to the (A) CK2 activity assay, (B) western blot analysis and (C) SA- $\beta$-gal assay. Values are presented as the mean \pm standard deviation of three independent experiments. The P-value was calculated by one-way analysis of variance followed by Tukey's honest significant difference post hoc test. ${ }^{\# \#} \mathrm{P}<0.01$ and ${ }^{\# \# \#} \mathrm{P}<0.001$ vs. negative control. ${ }^{* *} \mathrm{P}<0.01$ and ${ }^{* * *} \mathrm{P}<0.001$ vs. SCR. The difference between the negative control and SCR was not significant. siRNA, small interfering RNA; CK2, protein kinase casein kinase 2; TBB, 4,5,6,7-terabromo-2-azabenzimidazole; SA- $\beta$-gal, senescence-associated $\beta$-galactosidase; SCR, scrambled siRNA negative control.

effect of the peroxynitrite generator SIN-1, which is widely used to increase the expression and activity of HO-1 in several cell types, on TBB-induced chondrocyte senescence. In our previous study, it was reported that CK2 modulates 
A

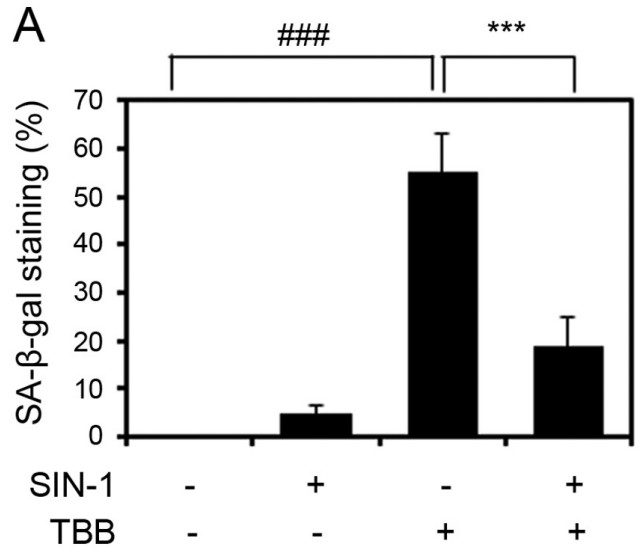

C

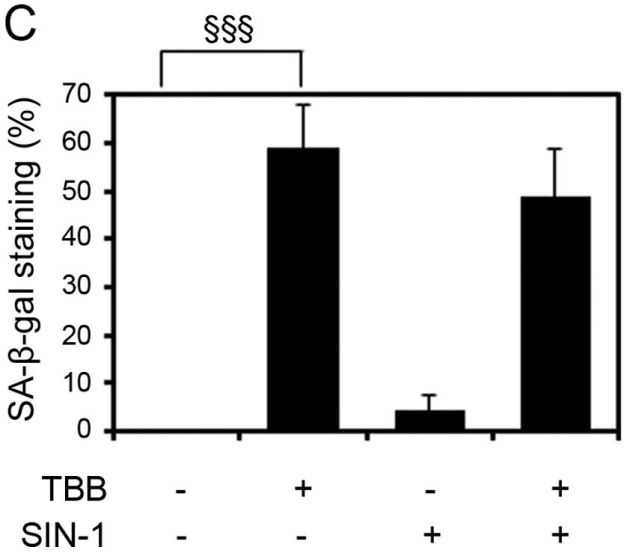

B

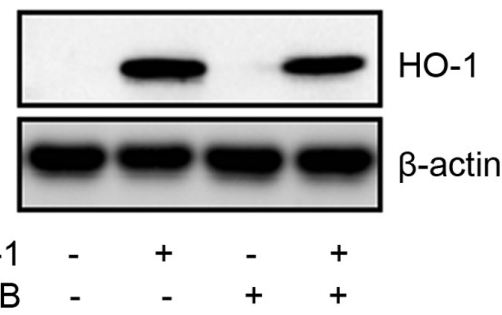

D

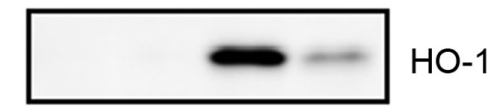

$\beta$-actin

Figure 4. Effect of SIN-1 on TBB-induced chondrocyte senescence. (A) SA- $\beta$-gal assay and (B) western blot analysis of chondrocytes pretreated with $200 \mu \mathrm{M}$ SIN-1 for $6 \mathrm{~h}$, stimulated with $50 \mu \mathrm{M}$ TBB, and incubated for $48 \mathrm{~h}$. ${ }^{\# \#} \mathrm{P}<0.001$ : negative control vs. TBB; ${ }^{* * *} \mathrm{P}<0.001$ : TBB vs. SIN-1 + TBB. P-values among the other groups were also analyzed (negative control vs. SIN-1: ns; negative control vs. SIN-1 + TBB: P<0.05; SIN-1 vs. TBB: P<0.001; SIN-1 vs. SIN-1 + TBB: $\mathrm{P}<0.05$ ). (C) SA- $\beta$-gal assay and (D) western blot analysis of chondrocytes stimulated with $50 \mu \mathrm{M}$ TBB for $6 \mathrm{~h}$, and incubated for another $42 \mathrm{~h}$ in the absence or presence of SIN-1 $(200 \mu \mathrm{M}) .{ }^{\S \S} \mathrm{P}<0.001$ : negative control vs. TBB. P-values among the other groups were also analyzed (negative control vs. SIN-1: ns; negative control vs. TBB + SIN-1: P<0.001; TBB vs. SIN-1: P<0.001; TBB vs. TBB + SIN-1: ns; SIN-1 vs. TBB + SIN-1: P<0.001). Values are presented as the mean \pm standard deviation of three independent experiments. P-values were calculated by one-way analysis of variance followed by Tukey's honest significant difference post hoc test. For western blotting, $\beta$-actin was used as an internal control. SIN-1, 3-morpholinosydnonimine hydrochloride; TBB, 4,5,6,7-terabromo-2-azabenzimidazole; SA- $\beta$-gal, senescence-associated $\beta$-galactosidase; ns, not significant; HO-1, heme oxygenase-1.

SIN-1-induced HO-1 expression in chondrocytes (23), and as a result it was hypothesized that TBB-induced chondrocyte senescence was caused by the inhibition of HO-1. In the present study, the chondrocytes were cultured for $6 \mathrm{~h}$ either in medium alone or in medium containing $200 \mu \mathrm{M} \mathrm{SIN}-1$ and stimulated with TBB $(50 \mu \mathrm{M})$ for $48 \mathrm{~h}$. As shown in Fig. 4A, pretreatment with SIN-1 suppressed TBB-induced cellular senescence, which resulted from the induction of the expression of $\mathrm{HO}-1$ by pretreatment with SIN-1 (Fig. 4B). However, when the cells were treated with SIN-1 following TBB, no significant reduction in SA- $\beta$-gal staining was observed (Fig. 4C). In addition, as expected, pretreatment with TBB inhibited the induction of HO-1 by SIN-1 (Fig. 4D), indicating that the effect of SIN-1 on the TBB-induced senescence of chondrocytes is associated with the expression of HO-1.

HO-1 inhibits TBB-induced cellular senescence in chondrocytes. Based on the above-mentioned results, the enhancement of chondrocyte senescence by TBB treatment appeared to be associated with the expression of HO-1, therefore, an HO-1-overexpression vector was used to determine whether
HO-1 directly modulates the senescence of TBB-treated chondrocytes. The chondrocytes were transfected with the HO-1 expression vector or control pcDNA vector and the protein overexpression of HO-1 was assessed by western blot analysis (Fig. 5A). As shown in Fig. 5B, the overexpression of HO-1 significantly reduced the senescence-specific SA- $\beta$-gal staining of TBB-treated chondrocytes. These results suggest that the forced overexpression of $\mathrm{HO}-1$ decreased the $\mathrm{CK} 2$ inhibition-mediated senescence of chondrocytes.

\section{Discussion}

Under physiological conditions, chondrocytes have rare proliferative properties and have a central role in the make up of articular cartilage, which contains an avascular cartilage-specific matrix (25). Chondrocytes produce and maintain extracellular matrix, which is composed of type II collagen and sulfated proteoglycans, by facilitating the cell-matrix interactions responsible for several of the functions of cartilage $(4,26)$. Although articular cartilage is hypoxic in nature, chondrocytes produce ROS to enable metabolism adapted to 
A

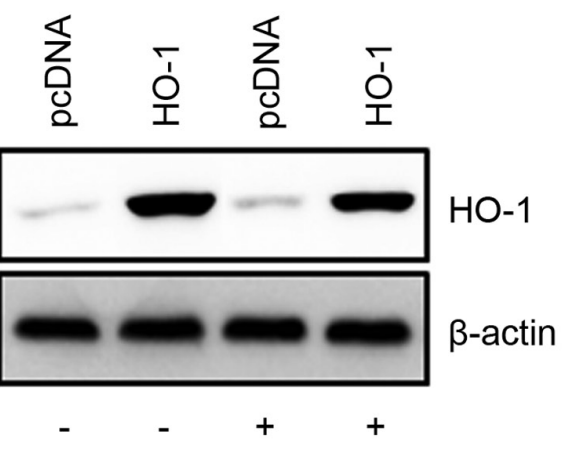

B

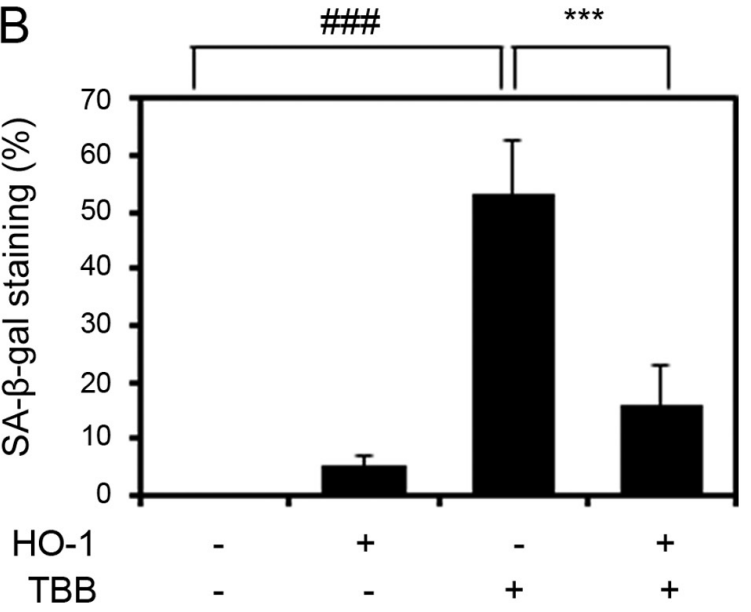

Figure 5. Effect of the overexpression of HO-1 on TBB-induced chondrocyte senescence. (A) Cells were subjected to western blot analysis to show the efficiency of HO-1 expression. $\beta$-actin was used as an internal control. (B) Cells were transfected with the HO-1 expression vector, incubated for $24 \mathrm{~h}$, stimulated with $50 \mu \mathrm{M}$ TBB for $48 \mathrm{~h}$, and subjected to a SA- $\beta$-gal assay. Values are presented as the mean \pm standard deviation of three independent experiments. P-values were calculated by one-way analysis of variance followed by Tukey's honest significant difference post hoc test. ${ }^{\# \#} \mathrm{P}<0.001$ : negative control vs. TBB, ${ }^{* * *} \mathrm{P}<0.001$ : TBB vs. HO- $1+\mathrm{TBB}$. P-values among other groups are also analyzed (negative control vs. HO-1: ns; negative control vs. HO-1 + TBB: ns; HO-1 vs. TBB: P<0.001; HO-1 vs. HO-1 + TBB: ns). TBB, 4,5,6,7-terabromo-2-azabenzimidazole; SA- $\beta$-gal, senescence-associated $\beta$-galactosidase; ns, not significant; HO-1, heme oxygenase-1.

anaerobic conditions, and is exposed to abnormal levels of ROS produced by immune cells under pathological conditions, including OA, rheumatoid arthritis and gout (27-29). Chondrocytes constitutively express well-coordinated antioxidant enzymes, including superoxide dismutase (SOD; cytosolic $\mathrm{Cu} / \mathrm{Zn}$ SOD and mitochondrial Mn SOD), glutathione peroxidase (GPX) and catalase (30,31).

Elevated levels of ROS and antioxidant depletion have been reported under pathological conditions, including those associated with inflammatory diseases (32). Oxidative stress can damage the components of the ECM by upregulating matrix metalloproteinases (MMPs) and inflammatory mediators, including inducible nitric oxide synthase and cyclooxygenase-2 $(33,34)$, and by doing so can cause the apoptosis and senescence of chondrocytes in articular cartilage and destroy articular cartilage $(9,13,35)$. Furthermore, age-related changes in the intracellular redox status of chondrocytes has been shown to be associated with the development of cell alterations $(36,37)$. Apoptotic chondro- cyte death is widely observed in degenerated cartilage of OA, and chondrocyte senescence may contribute to the reduction in chondrocyte numbers in articular cartilage; senescent chondrocytes accumulate with age and in articular cartilage of patients with OA $(8,11-13)$.

Unlike SOD, GPX, and catalase, HO-1 is adaptively induced to protect chondrocytes and cartilage against the destructive effects of oxidative stress (10). It has also been reported that HO-1 inhibits catabolic enzymes and inflammatory cytokines that may contribute to the pathogeneses of cartilage diseases (5), which suggests that HO-1 is a component of defense systems that protect articular cartilage $(27,38)$. In addition, it has been shown that HO-1 contributes significantly to protection against cartilage degeneration in humans (39). However, the mechanisms responsible for the regulation and activities of HO-1 in the contexts of chondrocyte maintenance, senescence and apoptosis in articular cartilage remain to be fully elucidated.

CK2 is critical in the control of cell proliferation, transformation and apoptosis (17-19), and reportedly, loss of CK2 activity is involved in chromatin structural alteration and changes in gene expression $(40,41)$. Therefore, it appears that CK2 can stimulate the phosphorylation of several proteins required for DNA replication and transcription $(15,42,43)$. In our previous study, it was reported that CK2 mediates the expression of HO-1 by phosphorylating and inducing the nuclear translocation of Nrf2 in chondrocytes exposed to oxidative stress (23). In the present study, it was found that the activity of CK2 is associated with the senescence of primary articular chondrocytes, and that the downregulation of CK2 induces cellular senescence by inhibiting the expression of HO-1. Furthermore, inhibition of CK2 activity, achieved using TBB (a specific CK2 inhibitor) or by silencing the CK2 gene, caused specific SA- $\beta$-gal staining of chondrocytes and altered the expression of type II collagen and $\beta$-catenin (phenotypic markers of chondrocyte differentiation). Subsequently, the present study investigated whether HO-1 is involved in TBB-induced chondrocyte senescence using the pharmacologic inducer (SIN-1) and by the transfection-induced overexpression of HO-1. Chondrocytes overexpressing HO-1 exhibited significant inhibition of TBB-induced cellular senescence, which provides evidence that the activity of $\mathrm{HO}-1$ is associated with the CK2 inhibition-mediated senescence of chondrocytes, and suggests that HO-1 may act to inhibit chondrocyte senescence.

However, reports indicate that $\mathrm{HO}-1$ has a dual role in pathological states, as high levels of HO-1 are frequently detected in pathological tissues $(44,45)$. In parallel, chondrocytes obtained by serial subculture exhibit passage number-dependent increases in cellular senescence and expression of HO-1 (unpublished data), which may reflect age-related changes in intracellular redox status during the development of cellular senescence. This observation encourages the suggestion that the level of HO-1 in articular cartilage may be a useful biomarker of the degree of articular cartilage degeneration. Based on the results obtained in the present study, it can be concluded that the activity of CK2 may act as an anti-aging factor by inducing the expression of HO-1 to counteract the effects of chondrocyte senescence in articular cartilage. 


\section{Acknowledgements}

Not applicable.

\section{Funding}

The present study was supported by the 2013 Specialization Project Research Grant Funded by Pusan National University.

\section{Availability of data and materials}

All the datasets generated and analysed during the present study are available from the corresponding author on reasonable request.

\section{Authors' contributions}

KMK, KK and YCP made contributions to the conception and design of the study. KMK, DHS and YCP performed the experiments. DHS, KK, and YCP performed the statistical analysis. DHS and YCP wrote and revised the manuscript. All authors read and approved the final manuscript.

\section{Ethics approval and consent to participate}

All animal experiments and protocols were approved by the Pusan National University Institutional Animal Care and Use Committee and performed in accordance with the institutional and national guidelines for the care and use of laboratory animals.

\section{Patient consent for publication}

Not applicable.

\section{Competing interests}

The authors declare that they have no competing interests.

\section{References}

1. Sandell LJ and Adler P: Developmental patterns of cartilage. Front Biosci 4: D731-D742, 1999.

2. DeLise AM, Fischer L and Tuan RS: Cellular interactions and signaling in cartilage development. Osteoarthritis Cartilage 8 : 309-334, 2000.

3. Goldring MB: The role of the chondrocyte in osteoarthritis. Arthritis Rheum 43: 1916-1926, 2000.

4. Roughley PJ: Articular cartilage and changes in arthritis: Noncollagenous proteins and proteoglycans in the extracellular matrix of cartilage. Arthritis Res 3: 342-347, 2001.

5. Guillén M, Megías J, Gomar F and Alcaraz M: Haem oxygenase-1 regulates catabolic and anabolic processes in osteoarthritic chondrocytes. J Pathol 214: 515-522, 2008.

6. Cawston T, Billington C, Cleaver C, Elliott S, Hui W, Koshy P, Shingleton B and Rowan A: The regulation of MMPs and TIMPs in cartilage turnover. Ann N Y Acad Sci 878: 120-129, 1999.

7. Kim HA and Song YW: Apoptotic chondrocyte death in rheumatoid arthritis. Arthritis Rheum 42: 1528-1537, 1999.

8. Aigner T and Kim HA: Apoptosis and cellular vitality: Issues in osteoarthritic cartilage degeneration. Arthritis Rheum 46 1986-1996, 2002.

9. Jallali N, Ridha H, Thrasivoulou C, Underwood C, Butler PE and Cowen T: Vulnerability to ROS-induced cell death in ageing articular cartilage: The role of antioxidant enzyme activity. Osteoarthritis Cartilage 13: 614-622, 2005.
10. Zwerina J, Tzima S, Hayer S, Redlich K, Hoffmann O, Hanslik-Schnabel B, Smolen JS, Kollias G and Schett G: Heme oxygenase $1(\mathrm{HO}-1)$ regulates osteoclastogenesis and bone resorption. FASEB J 19: 2011-2013, 2005.

11. Loeser RF: Aging and osteoarthritis: The role of chondrocyte senescence and aging changes in the cartilage matrix. Osteoarthritis Cartilage 17: 971-979, 2009.

12. Martin JA and Buckwalter JA: Aging, articular cartilage chondrocyte senescence and osteoarthritis. Biogerontology 3 : 257-264, 2002.

13. McCulloch K, Litherland GJ and Rai TS: Cellular senescence in osteoarthritis pathology. Aging Cell 16: 210-218, 2017.

14. Litchfield DW: Protein kinase CK2: Structure, regulation and role in cellular decisions of life and death. Biochem J 369: 1-15, 2003.

15. Meggio F and Pinna LA: One-thousand-and-one substrates of protein kinase CK2? FASEB J 17: 349-368, 2003.

16. Pinna LA: Casein kinase 2: An 'eminence grise' in cellular regulation? Biochim Biophys Acta 1054: 267-284, 1990.

17. Ahmed K, Gerber DA and Cochet C: Joining the cell survival squad: An emerging role for protein kinase CK2. Trends Cell Biol 12: 226-230, 2002

18. Faust RA, Gapany M, Tristani P, Davis A, Adams GL and Ahmed K: Elevated protein kinase CK2 activity in chromatin of head and neck tumors: Association with malignant transformation. Cancer Lett 101: 31-35, 1996.

19. Ahmad KA, Wang G, Unger G, Slaton J and Ahmed K: Protein kinase CK2 - a key suppressor of apoptosis. Adv Enzyme Regul 48: 179-187, 2008.

20. Drygin D, Ho CB, Omori M, Bliesath J, Proffitt C, Rice R, Siddiqui-Jain A, O'Brien S, Padgett C, Lim JK, et al: Protein kinase CK2 modulates IL-6 expression in inflammatory breast cancer. Biochem Biophys Res Commun 415: 163-167, 2011.

21. Yoon YM, Kim SJ, Oh CD, Ju JW, Song WK, Yoo YJ, Huh TL and Chun JS: Maintenance of differentiated phenotype of articular chondrocytes by protein kinase $\mathrm{C}$ and extracellular signal-regulated protein kinase. J Biol Chem 277: 8412-8420, 2002.

22. Lee SW, Song YS, Lee SY, Yoon YG, Lee SH, Park BS, Yun I, Choi H, Kim K, Chung WT, et al: Downregulation of protein kinase CK2 activity facilitates tumor necrosis factor- $\alpha$-mediated chondrocyte death through apoptosis and autophagy. PLoS One 6: e19163,2011.

23. Kim KM, Song JD, Chung HT and Park YC: Protein kinase CK2 mediates peroxynitrite-induced heme oxygenase-1 expression in articular chondrocytes. Int J Mol Med 29: 1039-1044, 2012.

24. Kim KM, Park SE, Lee MS, Kim K and Park YC: Induction of heme oxygenase-1 expression protects articular chondrocytes against cilostazol-induced cellular senescence. Int J Mol Med 34: 1335-1340, 2014.

25. Muir H: The chondrocyte, architect of cartilage. Biomechanics, structure, function and molecular biology of cartilage matrix macromolecules. BioEssays 17: 1039-1048, 1995.

26. Bau B, Gebhard PM, Haag J, Knorr T, Bartnik E and Aigner T: Relative messenger RNA expression profiling of collagenases and aggrecanases in human articular chondrocytes in vivo and in vitro. Arthritis Rheum 46: 2648-2657, 2002.

27. Henrotin YE, Bruckner P and Pujol JP: The role of reactive oxygen species in homeostasis and degradation of cartilage. Osteoarthritis Cartilage 11: 747-755, 2003.

28. Phillips DC, Dias HK, Kitas GD and Griffiths HR: Aberrant reactive oxygen and nitrogen species generation in rheumatoid arthritis (RA): Causes and consequences for immune function, cell survival, and therapeutic intervention. Antioxid Redox Signal 12: 743-785, 2010.

29. Martin WJ, Herst PM, Chia EW and Harper JL: Sesquiterpene dialdehydes inhibit MSU crystal-induced superoxide production by infiltrating neutrophils in an in vivo model of gouty inflammation. Free Radic Biol Med 47: 616-621, 2009.

30. Deahl ST II, Oberley LW, Oberley TD and Elwell JH: Immunohistochemical identification of superoxide dismutases, catalase, and glutathione-S-transferases in rat femora. J Bone Miner Res 7: 187-198, 1992.

31. Borsiczky B, Szabó Z, Jaberansari MT, Mack PP and Röth E: Activated PMNs lead to oxidative stress on chondrocytes: A study of swine knees. Acta Orthop Scand 74: 190-195, 2003.

32. Matés JM, Pérez-Gómez C and Núñez de Castro I: Antioxidant enzymes and human diseases. Clin Biochem 32: 595-603, 1999.

33. Stadler J, Stefanovic-Racic M, Billiar TR, Curran RD, McIntyre LA, Georgescu HI, Simmons RL and Evans CH: Articular chondrocytes synthesize nitric oxide in response to cytokines and lipopolysaccharide. J Immunol 147: 3915-3920, 1991 
34. Pelletier JP, McCollum R, DiBattista J, Loose LD, Cloutier JM and Martel-Pelletier J: Regulation of human normal and osteoarthritic chondrocyte interleukin-1 receptor by antirheumatic drugs. Arthritis Rheum 36: 1517-1527, 1993.

35. Henrotin Y, Kurz B and Aigner T: Oxygen and reactive oxygen species in cartilage degradation: Friends or foes? Osteoarthritis Cartilage 13: 643-654, 2005.

36. Carlo MD Jr and Loeser RF: Increased oxidative stress with aging reduces chondrocyte survival: Correlation with intracellular glutathione levels. Arthritis Rheum 48: 3419-3430, 2003.

37. Murray MM, Zurakowski D and Vrahas MS: The death of articular chondrocytes after intra-articular fracture in humans. J Trauma 56: 128-131, 2004.

38. Clérigues V, Guillén MI, Gomar F and Alcaraz MJ: Haem oxygenase- 1 counteracts the effects of interleukin- $1 \beta$ on inflammatory and senescence markers in cartilage-subchondral bone explants from osteoarthritic patients. Clin Sci (Lond) 122: 239-250, 2012

39. Fernández P, Guillén MI, Gomar F and Alcaraz MJ: Expression of heme oxygenase-1 and regulation by cytokines in human osteoarthritic chondrocytes. Biochem Pharmacol 66: 2049-2052, 2003.
40. Villeponteau B: The heterochromatin loss model of aging. Exp Gerontol 32: 383-394, 1997.

41. Yu S, Wang H, Davis A and Ahmed K: Consequences of CK2 signaling to the nuclear matrix. Mol Cell Biochem 227: 67-71, 2001.

42. Lorenz P, Ackermann K, Simoes-Wuest P and Pyerin W: Serum-stimulated cell cycle entry of fibroblasts requires undisturbed phosphorylation and non-phosphorylation interactions of the catalytic subunits of protein kinase CK2. FEBS Lett 448: 283-288, 1999.

43. St-Denis NA and Litchfield DW: Protein kinase CK2 in health and disease: From birth to death: the role of protein kinase CK2 in the regulation of cell proliferation and survival. Cell Mol Life Sci 66: 1817-1829, 2009.

44. Otterbein LE, Kolls JK, Mantell LL, Cook JL, Alam J and Choi AM: Exogenous administration of heme oxygenase-1 by gene transfer provides protection against hyperoxia-induced lung injury. J Clin Invest 103: 1047-1054, 1999.

45. Bauer M and Bauer I: Heme oxygenase-1: Redox regulation and role in the hepatic response to oxidative stress. Antioxid Redox Signal 4: 749-758, 2002. 